\title{
Triton-B Mediated One-Pot Multicomponent Synthesis of 3,5 Substituted Tetrahydro-2H-1,3,5-Thidiazine-2-Thiones
}

\author{
RAM KISHORE ${ }^{1}$, MONIKA KAMBOJ ${ }^{1 *}$ and MANISHA SHUKLA ${ }^{2}$ \\ 'Department of Applied Chemistry, Amity School of Applied Sciences, Amity University Uttar Pradesh, \\ Lucknow Campus, Lucknow-226028, U. P., India. \\ 2Department of Chemistry, BBDNITM, Lucknow-227105, U. P., India. \\ *Corresponding author E-mail: mkamboj@ @ko.amity.edu \\ http://dx.doi.org/10.13005/ojc/340627
}

(Received: June 30, 2018; Accepted: October 27, 2018)

\begin{abstract}
A new-fangled, proficient, one-pot multi component, intramolecular C-S bond formation reaction, mediated by phase transfer catalyst, Triton-B, is described in this paper. The reaction of alkyl/phenyl amines, $\mathrm{CS}_{2}$ and formaldehyde catalyzed via Triton-B resulted in formation of 3-(Alkyl or aryl methyl), 5-(Alkyl or aryl methyl) substituted tetrahydro-2H-1,3,5-thiadiazine-2-thiones compounds(1a-15a). These compounds (1a-15a) were characterized with the help of elemental analysis, IR, NMR and mass spectroscopic methods. The PTC mediated reactions require mild reaction condition and reduced time period for completion. The reaction is achieved at normal temperature under solvent free conditions with good yields and great selectivity. This methodology discourages the traditional synthesis method of inorganic base for such coupling reaction.
\end{abstract}

Keywords: Multi component, $\mathrm{CS}_{2}$, Triton-B, Phase transfer catalyst.

\section{INTRODUCTION}

3,5 substituted tetrahydro- $2 \mathrm{H}-1,3,5-$ thiadiazine-2-thiones (THTT), an important scaffold have found utility as chemicals for crop protection ${ }^{1}$, against soil nematodes ${ }^{2}$, antitumor drugs $^{3}$, precursors in organic synthesis ${ }^{4}$ and as antimicrobial agent. ${ }^{5}$ Recently dithiocarbamates developed as a novel class of prospective agrochemicals. ${ }^{6-9}$ Also they are potential pharmaceutical drugs against microbial infection $^{10}$, protozoa ${ }^{11}$, leprosy ${ }^{12}$, tubercular ${ }^{13}$, fungal, ${ }^{14}$ Leishmaniasis $^{15}$, Alzheimer's disease. ${ }^{16}$ Owing to ample of application, there is growth of well-planned methods for preparing 3-(Alkyl or aryl methyl), 5-(Alkyl or aryl methyl) substituted tetrahydro-2H-1,3,5-thiadiazine-2-thiones. New strategies for the synthesis of 3-(Alkyl or aryl methyl), 5-(Alkyl or aryl methyl) substituted tetrahydro-2H1,3,5-thiadiazine-2-thiones by using Triton-B, a phase transfer catalyst (PTC) delightfully supplement the conventional approach of cyclization by multicomponent reaction, ${ }^{17-18}$ based on inorganic base reactions. The inorganic base such as $\mathrm{NaOH}, \mathrm{KOH}, \mathrm{Na}_{2} \mathrm{CO}_{3}, \mathrm{H}_{2} \mathrm{O} / \mathrm{EtOH}$, uniform $\mathrm{H}_{2} \mathrm{O}$ catalyzed intra-molecular cyclization of amines, $\mathrm{CS}_{2}$ and formaldehyde that were produce in situ or

This is an Open Access article licensed under a Creative Commons license: Attribution 4.0 International (CC- BY). Published by Oriental Scientific Publishing Company @ 2018 
resynthesized are the traditional recur methods ${ }^{19-}$ 20 (Previous work) "Scheme 1". On the one hand, current effort is PTC-mediated prolific methodology to prepare THTT (Current work) "Scheme 1". The adeptness of this method is (i) excess amounts of toxic reagents are avoided in this scheme that adversely affects the health. (ii) cost of PTC is low (iii) high yield (iv) reduced reaction time. (v) Triton-B is recovered from the reaction mixture by filtration ${ }^{21}$. These factors discourage the use of inorganic base for such coupling reactions. This simple procedure with mild reaction conditions are safer and more sustainable practices for effective coupling reaction mediated by Trimethylbenzyl ammonium hydroxide (Triton-B) without using any inorganic base ${ }^{22}$, "Scheme 1"

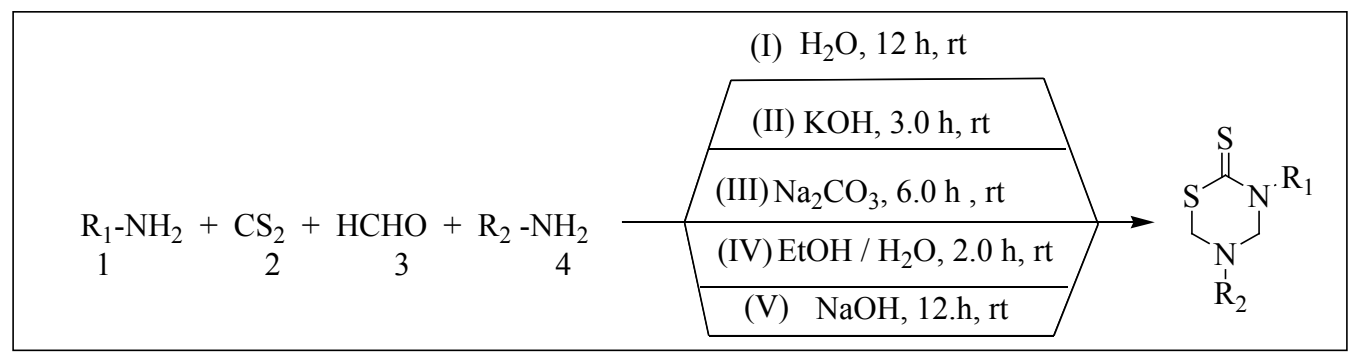

Previous Work

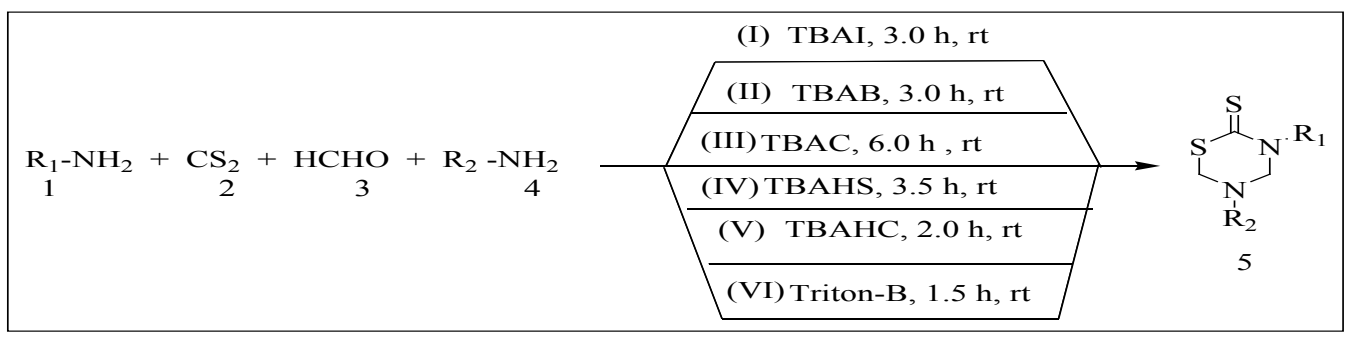

Current Work

Scheme 1. Comparison of previous works with current reported work

\section{MATERIALS AND METHODS}

All the chemicals were of AR grade. Reactions were done under an envelope of Ar gas. Bomem MB-104-FTIR spectrophotometer was used for recording Infra Red Spectra in the range 4000-200 $\mathrm{cm}^{-1}$. The AC-400F-NMR spectrometer, with Me4Si as internal standard was used for recording ${ }^{1} \mathrm{HNMR}$ spectra at $400 \mathrm{MHz}$. The investigation of elements were conveyed with the help of a Carlo-Erba EA $1110-C N N O-S$ analyzer. There is good covenant between observed and calculated values.

\section{General Method for the synthesis of compounds (1a-15a) using Triton-B:}

At room temperature $1.0 \mathrm{mmol}$ of primary amine (1) and $10.0 \mathrm{mmol} \mathrm{CS}_{2}$ (2) and $2.5 \mathrm{mmol}$ formaldehyde (3) was stirred for 0.25 hours. Then to this solution, $1.5 \mathrm{mmol}$ Triton-B was added and stirred for 0.25 hours. Afterward $1.0 \mathrm{mmol}$ amine (4) was added at room temperature for $2 \mathrm{~h}$, the stirring of reaction mixture was continued.
The advancement of reaction was observed through Thin Layer Chromatography. After accomplishment of reaction, $50 \mathrm{~mL}$ water was added and thrice treated with $\mathrm{CH}_{3} \mathrm{COOC}_{2} \mathrm{H}_{5}$ (20 mL each). The crude raw product was obtained by concentrating ether layer under low pressure which was further refined by silica gel (100-200 mesh) column chromatography by using eluent 50\% (EtOAc : Hexane) to afford pure product.

\section{RESULTS AND DISCUSSION}

In the present work, series of substituted THTT (1a-15a) are synthesized by Triton B mediated one-pot multicomponent reaction, a novel protocol (Scheme 2). Earlier THTT was produced from amines, $\mathrm{CS}_{2}$, formaldehyde using inorganic base $\mathrm{e}^{23-25}$. A number of PTC is frequently used for the synthesis of $\mathrm{THTT}^{26}$. On comparing the reaction condition it was comprehended that by using Triton-B, \% yields of preferred product is improved than other types of PTC.17 Triton-B give 95\% yield of THTT 
A range of 10 amines (aliphatic, alicyclic, heterocyclic, aromatic) and formaldehyde using Triton-B/CS $\mathrm{CS}_{2}$ method at r.t led to formation of product THTT in excellent yields, "Table 1". It has been observed that electron donating group in $\mathrm{R}_{1} \mathrm{NH}_{2}$ or $\mathrm{R}_{2} \mathrm{NH}_{2}$ gives corresponding products in good amount. These compounds were characterized by spectroscopic techniques (Fig 1-3). Proposed reaction and Mechanisms shown in "Scheme-2".
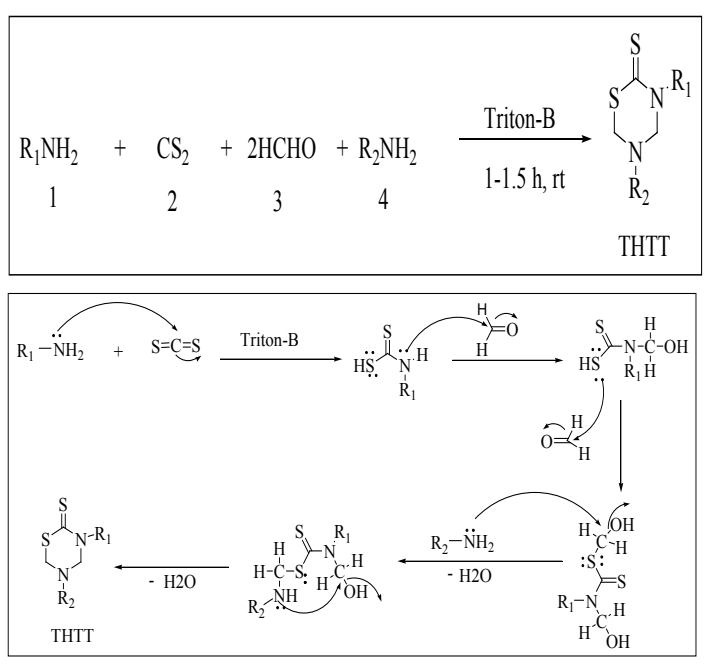

Scheme 2. Proposed Reaction and Mechanism
Spectral Data of selected synthesized dithio -carbamates (1a, 1b, 1c):

3,5-diethyl-1,3,5-thiadiazinane-2-thione (1a)

Yield 95\%; Off white solid, M.P. 104- $109^{\circ} \mathrm{C}$; ${ }^{1} \mathrm{H}$ NMR $400 \mathrm{MHz}\left(\mathrm{CDCl}_{3}\right): \delta 4.407$ (s, 2H), 4.346 (s, 2H), 4.028 (q, J = 7.2 Hz, 2H), 2.823 (q, J = 7.2 $\mathrm{Hz}, 2 \mathrm{H}), 1.232$ (t, J = 7.2 Hz, 3H), 1.155 (t, J = 7.2 Hz, $3 \mathrm{H}) .{ }^{13} \mathrm{C}$ NMR $400 \mathrm{MHz}\left(\mathrm{CDCl}_{3}\right): \delta 11.634,12.758$, 44.474, 46.951, 57.508, 68.998, 191.114. MS (ESI): $\mathrm{m} / \mathrm{z}=191.06[\mathrm{M}]+$. Anal. Calculated for $\mathrm{C}_{7} \mathrm{H}_{14} \mathrm{~N}_{2} \mathrm{~S}_{2}$ : 190.06: C, 44.17; H, 7.41; N, 14.72; S, 33.69. Found: C, 43.95; H, 7.17; N, 14.52; S, 33.48\%.

\section{3,5-bis(4-chlorobenzyl)-1,3,5-thiadiazinane-2- thione (2a)}

Yield 94\%; Off white solid, M.P. $105-110^{\circ} \mathrm{C}$; ${ }^{1} \mathrm{H}$ NMR $400 \mathrm{MHz}\left(\mathrm{CDCl}_{3}\right): \delta$ 7.364-7.335 (m, 2H), $7.305(\mathrm{~d}, \mathrm{~J}=8.4 \mathrm{~Hz}, 2 \mathrm{H}), 7.236(\mathrm{~d}, \mathrm{~J}=8.4 \mathrm{~Hz}$, 2H), 7.024 (d, J = 8.4 Hz, 2H), 5.254 (s, 2H), 4.356 (s, 2H), 4.269 (s, 2H), 3.718 (s, 2H). MS (ESI): m/z $=383.09[\mathrm{M}]+$. Anal. Calculated for $\mathrm{C}_{17} \mathrm{H}_{16} \mathrm{Cl}_{2} \mathrm{~N}_{2} \mathrm{~S}_{2}: \mathrm{C}$, 53.26; H, 4.21; Cl, 18.50; N, 7.31; S, 16.73. Found C, $53.01 ; \mathrm{H}, 4.11 ; \mathrm{Cl}, 18.25 ; \mathrm{N}, 7.11 ; \mathrm{S}, 16.52 \%$.

Table 1: Effect of Substituents on THTT formation

\begin{tabular}{|c|c|c|c|c|c|}
\hline Compound & $\mathrm{R}_{1}$ & $\mathrm{R}_{2}$ & Molecular Formula & Time (h) & Yield\% \\
\hline $1 a$ & $\begin{array}{c}\mathrm{C}_{2} \mathrm{H}_{5} \\
1\end{array}$ & $\begin{array}{c}\mathrm{C}_{2} \mathrm{H}_{5} \\
95\end{array}$ & $\mathrm{C}_{7} \mathrm{H}_{14} \mathrm{~N}_{2} \mathrm{~S}_{2}$ & & \\
\hline $2 a$ & $4-\mathrm{Cl}-\mathrm{PhCH}_{2}$ & $4-\mathrm{Cl}-\mathrm{PhCH}_{2}$ & $\mathrm{C}_{17} \mathrm{H}_{16} \mathrm{Cl}_{2} \mathrm{~N}_{2} \mathrm{~S}_{2}$ & 1 & 94 \\
\hline $3 a$ & $\mathrm{C}_{2} \mathrm{H}_{5}$ & 2-MeO- $\mathrm{PhCH}_{2}$ & $\mathrm{C}_{13} \mathrm{H}_{18} \mathrm{ON}_{2} \mathrm{~S}_{2}$ & 1 & 92 \\
\hline $4 a$ & $\mathrm{C}_{7} \mathrm{H}_{15}$ & $3-\mathrm{CF}_{3}-\mathrm{PhCH}_{2}$ & $\mathrm{C}_{18} \mathrm{H}_{25} \mathrm{~F}_{3} \mathrm{~N}_{2} \mathrm{~S}_{2}$ & 1 & 94 \\
\hline $5 a$ & $\mathrm{Ph}$ & $\mathrm{Ph}$ & $\mathrm{C}_{15} \mathrm{H}_{14} \mathrm{~N}_{2} \mathrm{~S}_{2}$ & 1.5 & 95 \\
\hline $6 a$ & $3-\mathrm{Cl}-\mathrm{PhCH}_{2}$ & $4-\mathrm{Cl}-\mathrm{PhCH}_{2}$ & $\mathrm{C}_{17} \mathrm{H}_{16} \mathrm{Cl}_{2} \mathrm{~N}_{2} \mathrm{~S}_{2}$ & 1 & 93 \\
\hline $7 a$ & 2-F-PhCH ${ }_{2}$ & $2-\mathrm{F}-\mathrm{PhCH}_{2}$ & $\mathrm{C}_{17} \mathrm{H}_{16} \mathrm{~F}_{2} \mathrm{~N}_{2} \mathrm{~S}_{2}$ & 1 & 93 \\
\hline $8 a$ & $\mathrm{PhC}_{4} \mathrm{H}_{8}$ & $4-\mathrm{CF}_{3}-\mathrm{PhCH}_{2}$ & $\mathrm{C}_{21} \mathrm{H}_{23} \mathrm{~F}_{3} \mathrm{~N}_{2} \mathrm{~S}_{2}$ & 1 & 90 \\
\hline $9 a$ & $\mathrm{C}_{2} \mathrm{H}_{5}$ & $2-\mathrm{F}-\mathrm{PhCH}_{2}$ & $\mathrm{C}_{12} \mathrm{H}_{15} \mathrm{FN}_{2} \mathrm{~S}_{2}$ & 1 & 94 \\
\hline $10 a$ & 2-F-PhCH & $4-\mathrm{Cl}-\mathrm{PhCH}_{2}$ & $\mathrm{C}_{17} \mathrm{H}_{16} \mathrm{CIFN}_{2} \mathrm{~S}_{2}$ & 1 & 90 \\
\hline $11 a$ & $\mathrm{C}_{2} \mathrm{H}_{5}$ & $\mathrm{C}_{4} \mathrm{H}_{9} \mathrm{O}$ & $\mathrm{C}_{9} \mathrm{H}_{18} \mathrm{ON}_{2} \mathrm{~S}_{2}$ & 1.5 & 92 \\
\hline $12 a$ & $\mathrm{PhC}_{4} \mathrm{H}_{8}$ & $\mathrm{PhC}_{4} \mathrm{H}_{8}$ & $\mathrm{C}_{23} \mathrm{H}_{30} \mathrm{~N}_{2} \mathrm{~S}_{2}$ & 1.5 & 93 \\
\hline $13 a$ & $\mathrm{C}_{5} \mathrm{H}_{11}$ & $\mathrm{C} 5 \mathrm{H} 11$ & $\mathrm{C}_{13} \mathrm{H}_{26} \mathrm{~N}_{2} \mathrm{~S}_{2}$ & 1.5 & 90 \\
\hline $14 a$ & $\mathrm{C}_{4} \mathrm{H}_{9}$ & $\mathrm{PhC}_{4} \mathrm{H}_{8}$ & $\mathrm{C}_{17} \mathrm{H}_{26} \mathrm{~N}_{2} \mathrm{~S}_{2}$ & 1.5 & 91 \\
\hline $15 a$ & $\mathrm{C}_{7} \mathrm{H}_{15}$ & $\mathrm{C}_{7} \mathrm{H}_{15}$ & $\mathrm{C}_{17} \mathrm{H}_{34} \mathrm{~N}_{2} \mathrm{~S}_{2}$ & 1 & 93 \\
\hline
\end{tabular}

$R_{1}$ and $R_{2}=1.0 \mathrm{mmol}$ each 


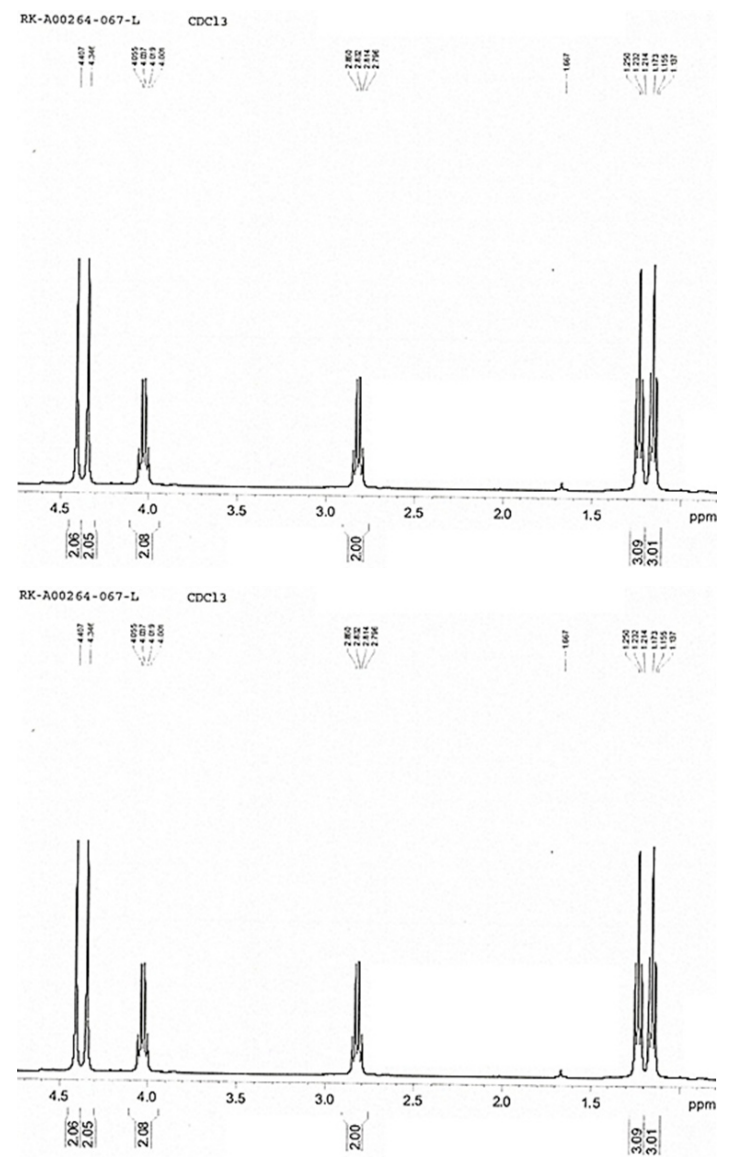

Fig. 1 (a). ${ }^{1}$ HNMR spectra of 3,5-diethyl-1,3,5-thiadiazinane-2-thione 2X-200264-079-U COC13

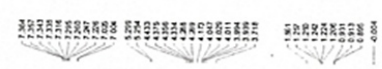

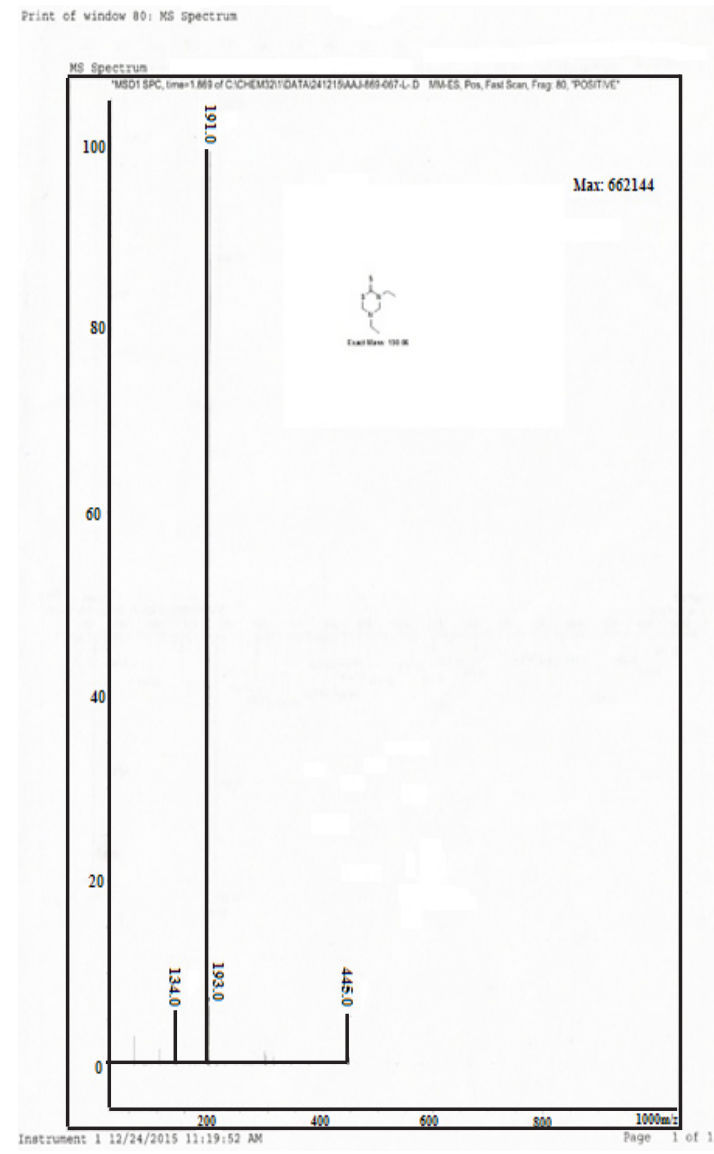

Fig 1(b). Mass spectra of 3,5-diethyl-1,3,5-thiadiazinane-2-thione 2x-A00264-079-v COC13
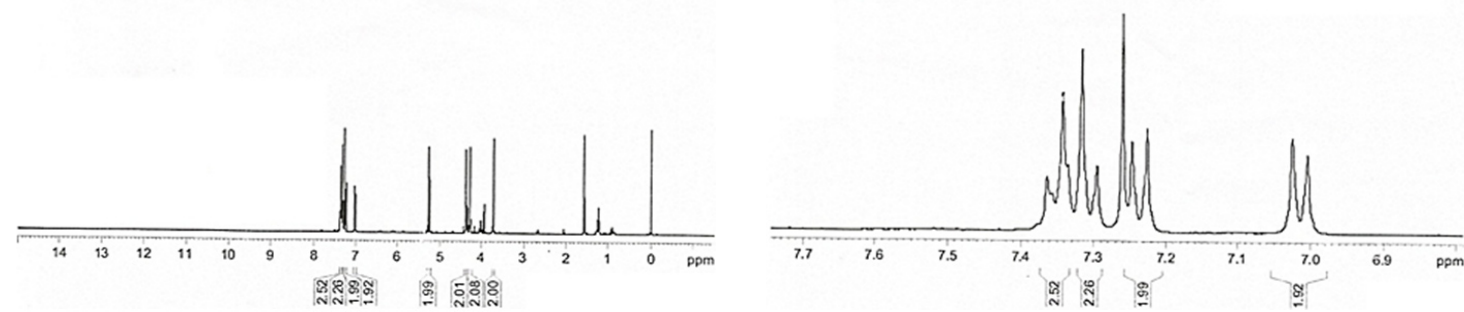

Fig. 2 (a). 'HNMR spectra of 3,5-bis(4-chlorobenzyl)-1,3,5-thiadiazinane-2-thione

3-(2-methoxybenzyl)-5-ethyl-1,3,5-thiadiazinane2-thione (3a)

Yield 92\%; Off white solid, M.P. 106-110 $\mathrm{C}$; ${ }^{1} \mathrm{H}$ NMR $400 \mathrm{MHz}\left(\mathrm{CDCl}_{3}\right): \delta 7.521(\mathrm{~d}, \mathrm{~J}=7.6 \mathrm{~Hz}$, $1 \mathrm{H}), 7.298(\mathrm{t}, \mathrm{J}=6.4 \mathrm{~Hz}, 1 \mathrm{H}), 6.952(\mathrm{t}, \mathrm{J}=7.2 \mathrm{~Hz}$, $1 \mathrm{H}), 6.893(\mathrm{~d}, \mathrm{~J}=8.0 \mathrm{~Hz}, 1 \mathrm{H}), 5.375(\mathrm{~s}, 2 \mathrm{H}), 4.411$ (s, 2H), 4.333 (s, 2H), 3.852 (s, 3H), 2.714 (q, J = 7.2
$\mathrm{Hz}, 2 \mathrm{H}), 0.943(\mathrm{t}, \mathrm{J}=7.2 \mathrm{~Hz}, 3 \mathrm{H}) \cdot{ }^{13} \mathrm{C}$ NMR $400 \mathrm{MHz}$ $\left(\mathrm{CDCl}_{3}\right): \delta 12.568,44.521,48.215,55.539,57.915$, $68.453,110.539,121.072,123.394,129.392$, 130.369, 157.471, 192.702. MS (ESI): $\mathrm{m} / \mathrm{z}=282.09$ $[\mathrm{M}]+$. Anal. Calculated for $\mathrm{C}_{13} \mathrm{H}_{18} \mathrm{ON}_{2} \mathrm{~S}_{2}: \mathrm{C}, 55.29 ; \mathrm{H}$, 6.42; N, 9.92; O, 5.67; S, 22.71. Found C, 55.03; H, $6.32 ; \mathrm{N}, 9.82 ; \mathrm{O}, 5.67 ; \mathrm{S}, 22.61 \%$. 

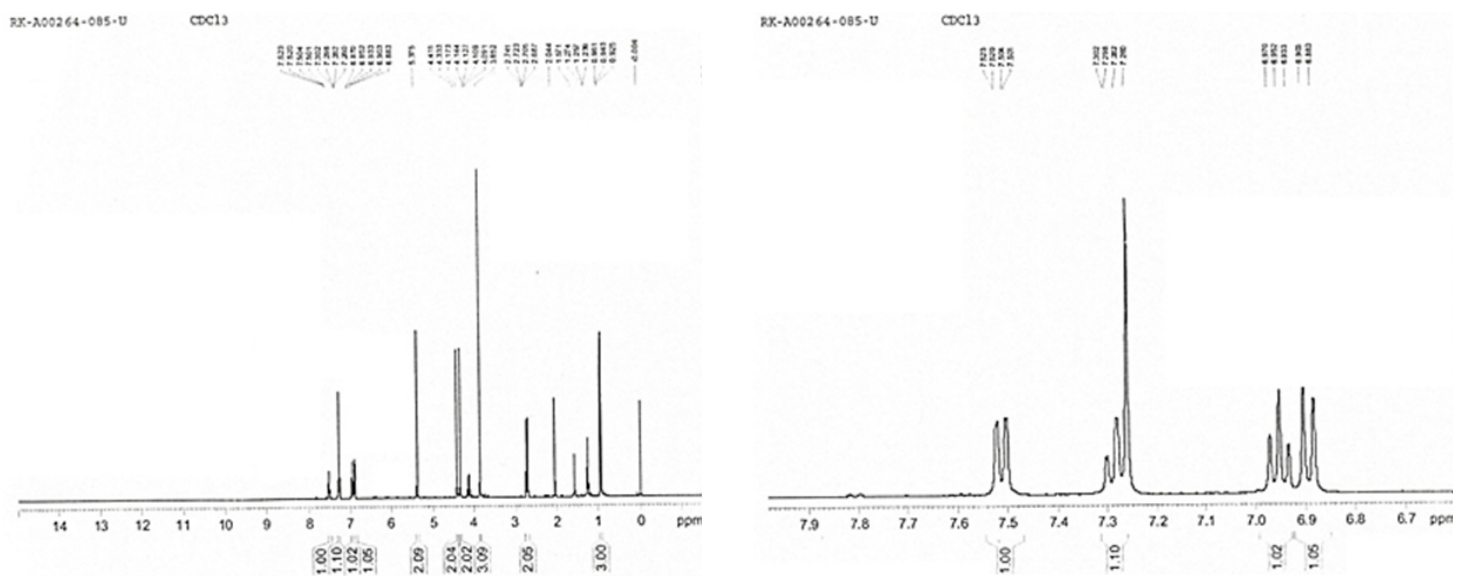

Fig. 3(a). ${ }^{1}$ HNMR spectra of 3-(2-methoxybenzyl)-5-ethyl-1,3,5-thiadiazinane-2-thione

CONCLUSION

We have developed highly efficient solventfree one-pot multicomponent coupling of various amines with formaldehyde via $\mathrm{CS}_{2} /$ Benzyl tri-methyl ammonium hydroxide system. This procedure gives corresponding products in good and excellent yield. Also, this procedure has mild reaction conditions, requires shorten time period and has environmental acceptability. This synthetic route thereby offers a more convenient approach for formation of C-S bonds.

\section{ACKNOWLEGEMENT}

Ram Kishore and Monika Kamboj appreciative the authorities of Amity University, Lucknow Campus, for their perpetual inspiration.

\section{REFERENCES}

1. Lamberth, C. J. Sulphur Chem., 2004, 25, 39-62.

2. Easton, A. ; Guven, K. ; de Pomerai, D.I. J. Biochem. Mol. Toxicol., 2001, 15, 15-25.

3. a) Cao, S.L.; Feng, Y.P.; Jiang, Y.Y.; Liu, S.Y.; Ding, G.Y.; Li, R.T. Bioorg. Med. Chem. Lett., 2005, 15, 1915-1917. (b) Cao, S. L. ; Han, Y. ; Yuan, C.Z. ; Wang, Y. ; Xiahou, Z.K. ; Liao, J. ; Gao, R.T.; Mao, B.B. ; Zhao, B.L. ; Li, Z. F. ; Xu, X. Eur J. Med. Chem., 2013, 64, 401-409.

4. Hassan, E.A.; Zayed, S.E. Phosphorus Sulfur Silicon Relat. Elem., 2014, 189, 300-323.

5. Ozcelik, A.B. ; Yilmaz, G. ; ozkan, S.; Ersan, S. Rev. Roum. Chim., 2015, 60, 1059-1064.

6. Haendel, M. A.; Tilton, F.; Bailey, G.S.; Tanguay, R.L. Toxicol. Sci., 2004, 81, 390-400.

7. Jardim, A.N.O.; Mello, D.C.; Brito, A.P.; Voet, H.V.; Boon, P.E.; Caldas, E.D. Food Chem. Toxicol., 2018, 118, 317-327.

8. Eng, G.; Song, X.; Duong, Q.; Strickman, D. ; Glass, J. ; May, L. Appl. Organomet. Chem., 2003, 17, 218-225.

9. Rogachev, I.; Kampel, V.; Gusis, V.; Cohen, N.; Gressel, J.; Warshawsky, A. Pest. Biochem. Physiol., 1998, 60, 133-145.
10. Guzel, O.; Salman, A. Bioorg. Med. Chem., 2006, 14, 7804-7815.

11. Ochoa, C.; Pérez, E.; Pérez, R.; Suárez, M.; Ochoa, E.; Rodríguez, H.; Gómez Barrio, A.; Muelas, S.; Nogal, J.J.; Martínez, R.A. Arzneim.-Forsch., 1999, 49,764-769. (b) Coro, J.; Atherton, R.; Little, S.; Wharton, H.; Yardley, V.; Alvarez, A. Jr.; Súarez, M.; Pérez, R.; Rodríguez, H. Alkyl-linked bis-THTT derivatives as potent in vitro trypanocidal agents. Bioorg. Med. Chem. Lett., 2006,16, 1312-1315.

12. Marakov, V.; Riabova, O.B.; Yuschenko, A.; Urlyapova, N.; Daudova, A.; Ziplef, P.F.; Mollmann, U. J. Antimicrob. Chemother., 2006, 57, 1134-1138.

13. Byrne, S.T.; Gu, P.; Zhou, J.; Denkin, S.M.; Chong, C.; Sullivan, D.; Liu, J.O.; Zhang, Y. Antimicrob. Agents Chemother., 2007, 51, 124495-124497.

14. Zou, Y.; Yu, S.; Li, R.; Zhao, Q.; Li, X.; Wu, M.; Huang, T.; Chai, X.; Hu, H.; Wu, Q. Euro J. Med. Chem., 2014, 74, 366-374.

15. Monzote, L.; Montalvo, A.M.; Fonseca, L.; Pérez, R.; Suárez, M.; Rodríguez, H. 
Arzneim.-Forsch., 2005, 55, 232-8.

16. Rehman,A. ; Nafeesa, K. ; Abbasi, M.A.; Siddiqui, S.Z. ;Rasool, S. ;Shah, S.A.A. ;Ashraf, M. ; Cogent Chemistry., 2018, 4, 1-15.

17. Zaidi, S.; Chaturvedi, A.K.; Singh, N.; Chaturvedia, D. Curr. Chem. Lett., 2017, 6, 143-150.

18. Kishore, R.; Kamboj, M. World j. Pharm. Res., 2018, 7, 1098-1109.

19. Katiyar, D.; Tiwari, V.K.; Tripathi, R.P.; Srivastava, A.; Chaturvedi, V.; Srivastava, R.; Srivastava, B.S. Bioorg. Med. Chem., 2003, 11, 4369-4375.
20. Ertan, M. ; Bilgin, A.A.; Palaska, E.; Yulug, N. Arzneim.-Forsch., 1992, 42, 160-163.

21. Chaturvedi, D.; Chaturvedi, A.K.;Mishra, N.;Mishra, V. Org. Chem. International., 2012, 2012,1 - 4.

22. Chaturvedi, D.; Zaidi, S. ; Chaturvedi, A.K.;Vaid,S. ;Saxena, A.K. Indian J Chem., 2016, 55B 1019-1025.

23. Ertan, M.; Ayyildiz, H.G.;Yulug, N. Arzneim.Forsch., 1991, 41,1182-1185.

24. Sharma, S. Synthesis., 1978, 11, 803-820.

25. Pascual, R. M. Synlett., 2015, 26, 1776-1777.

26. Saeed, S. Cogent Chem., 2015, 1,1-10. 